\title{
Recent Advancements in Toxin and Antitoxin Systems Involved in Bacterial Programmed Cell Death
}

\author{
Ming-xi Hu, Xiao Zhang, Er-li Li, and Yong-Jun Feng \\ School of Life Science, Beijing Institute of Technology, Beijing 100086, China \\ Correspondence should be addressed to Yong-Jun Feng, fengyj@bit.edu.cn \\ Received 8 June 2010; Revised 14 October 2010; Accepted 21 November 2010 \\ Academic Editor: Ingolf Figved Nes
}

Copyright () 2010 Ming-xi Hu et al. This is an open access article distributed under the Creative Commons Attribution License, which permits unrestricted use, distribution, and reproduction in any medium, provided the original work is properly cited.

Programmed cell death (PCD) systems have been extensively studied for their significant role in a variety of biological processes in eukaryotic organisms. Recently, more and more researches have revealed the existence of similar systems employed by bacteria in response to various environmental stresses. This paper summarized the recent researching advancements in toxin/antitoxin systems located on plasmids or chromosomes and their regulatory roles in bacterial PCD. The most studied yet disputed mazEF system was discussed in depth, and possible roles and status of such a special bacterial death and TA systems were also reviewed from the ecological and evolutionary perspectives.

\section{Introduction}

While the mechanism of autophagy and apoptosis in multicellular eukaryotes has been decoded progressively, many recent researches have suggested that simple prokaryotes like bacteria can also activate particular programmed cell death (PCD), allowing them to survive from the environmental stresses as nutrients deprivation, antibiotics, and so forth. Bacterial PCD has been observed in many bacteria species, including Escherichia, Staphylococcus, Pseudomonas, and Bacillus [1]. For eukaryotes, apoptosis plays an important role in embryonic development, immune tolerance and stability, cancer, inflammation, natural regeneration of cells, and other physiological or pathological processes [2]. But for prokaryotes, in essence, PCD is a kind of altruistic act that provides a way to survive the environmental stresses at the expense of some of its cells. This paper will discuss the recent advancement in toxin-antitoxin systems employed by bacteria in their PCD processes and the roles of bacterial PCD from the ecological and evolutionary perspectives.

\section{PCD Regulated by Toxin and Antitoxin Systems}

2.1. Toxin-Antitoxin (TA) Systems. For most eubacteria and archaea, the most common mechanism involved in bacterial
PCD is the toxin-antitoxin (TA) system. TA systems often include a stable toxin and a labile antitoxin; the former always exists as a stable protein, while the latter is either a protein or an untranslated antisense RNA species. In addition, the antitoxin gene often locates upstream of the toxin gene, usually overlapping or being separated by a small intergenic region, so that TA genes are cotranscribed and, in most cases, cotranslated. The expression of TA operon is autoregulated by antitoxin at the transcription level, while toxin acts as a corepressor [3]. TA systems are often divided into two types [4]: if the toxin is neutralized by an antisense RNA, the system is known as type I [5]; if the toxin is neutralized by avid binding of the partner antitoxin protein, the system is known as type II [6]. Meanwhile, an entirely new TA system has been defined recently, which functions via a novel protein-RNA mechanism [7]. During investigation on strategies used by bacteria in resisting bacteriophage infection, the ToxIN abortive infection (Abi) system is demonstrated as a new TA pair, with ToxN inhibiting bacterial growth and the tandem-repeat ToxI RNA antitoxin counteracting the toxicity. Noticeably, the ToxI RNA does not act like type I antitoxin, but is predicted to interact directly with ToxN and inhibit its toxicity.

Recent computational analyses have shown that TA systems are widely presented in eubacterial and archae chromosomes, yet their localizations are quite varied [8]. 
Based on massive amount of data, researchers have shown that type II TA systems have an extensive horizontal mobility [6]; while type I TA systems are not prone to transfer, but evolved from lineage-specific duplications [9]. Some TA systems are localized within exogenous DNA islands such as phages, transposons, and superintegrons; while others, like mazEF from E. coli $\mathrm{K}-12$, are settled in the regular positions of the chromosomes [10].

2.2. TA Systems Located on Plasmids. The $c c d$ (control cell death) system located on the E. coli $\mathrm{F}$ plasmid is the first TA system discovered [11]. The coexpressed gene products encoded by the $c c d$ operon are an unstable antitoxin CcdA that is degraded by the Lon ATP-dependent protease and a toxin CcdB that targets the DNA gyrase (an essential type II topoisomerase) $[12,13]$. Under normal growth conditions, the toxic activity of CcdB is inhibited by CcdA by forming a tightly binding iso-dimer [14]. But once the plasmid is lost, the short-lived CcdA will not be able to replenish by the de novo synthesis, leaving the long-lived toxin alone in the cytoplasm. As a result, DNA-gyrase complexes are trapped by CcdB, leading to eventually cell death [15]. Related investigations have found that plasmids R1/R100, P1, RK2/RP4, and RSF1010 also possess such type of TA systems, where the toxins interact with DNA helicase [16].

$\mathrm{Kid} / \mathrm{Kis}$ module on E. coli plasmid R1 belongs to another kind of TA systems, where toxin plays a role in cleaving RNA and inhibiting protein synthesis [17]. Kid and Kis are the stable toxin and unstable antitoxin encoded by the parD operon of plasmid R1 [18]. The parD operon is regulated by Kis and Kid coordinately [19]. Kid is a specific endoribonuclease to cleave RNA preferentially at the $5^{\prime}$ side of the adenosine residue in the nucleotide sequence $5^{\prime}$-UA(A/C)-3' of single-stranded regions. Kis autoregulates parD transcription to a limited extent through its $\mathrm{N}$-terminal region [20].

As the genetic units outside the chromosome, plasmids can self-replicate and maintain genetic stability of bacteria. In fact, they are likely to carry essential structural or functional gene sequences that the chromosome genome does not encode. Therefore, it is particularly important for the host to guarantee the stability of these plasmids. Many studies have showed that plasmids tend to have a core area to ensure their replication stability and conjugation transfer. During cell division, plasmid-specified partition proteins are activated. This process is directed by the plasmid itself. Because antitoxin is degraded continuously, the daughter cells that fail to acquire a plasmid copy will result in selective killing or growth impairment $[21,22]$.

As a suicidal system, TA loci only exhibit functions inside cells. This is obviously different from enterobactin or antibiotics, as they are always secreted into extracellular medium to reveal their bioactivity. Most of the wellcharacterized TA systems resided in plasmids are shown in Table 1.

2.3. TA Systems Located on Bacterial Chromosomes. TA systems are also abundant in chromosomes (Table 2) and show more complex phylogenetic patterns. In E. coli, there are several pairs of TA modules, including mazEF [23, 24], chpBIK [25], relBE [26, 27], yefM-yoeB [28, 29], and dinJyafQ [30] at least. Schmidt et al. [31] found that prlF and yhaV encode a new toxin-antitoxin system in E. coli. As homologs of MazE and RelE, respectively, PrlF and YhaV provide an evolutionary connection between the two bestcharacterized E. coli TA systems (mazEF and relEB). Besides the TA systems described in Table 2, three new chromosomal TA systems Rv1246c-Rv1247c, Rv2865-Rv2866, and Rv3357Rv3358, named as RelBE, RelFG, and RelJK, respectively, were identified in Mycobacterium tuberculosis recently [32].

While most TA modules conform to the characteristic cassette, in which the antitoxin gene precedes the toxin gene as mentioned above, there are also examples that the gene order is reversed, or the product of a third gene is implicated [21]. A recent study by Nariya and Inouye demonstrated that Myxococcus xanthus has a solitary mazF gene that lacks a cotranscribed antitoxin gene mazE [33]. The deletion of $m a z F$ causes elimination of the obligatory cell death during development, resulting in dramatic reduction in spore formation. Surprisingly, a key developmental regulator, $\mathrm{MrpC}$, is discovered to function as a MazF antitoxin and a $m a z F$ transcription activator. The transcription of $m r p C$ and mazF is negatively regulated via $\mathrm{MrpC}$ phosphorylation by a Ser/Thr kinase cascade.

In conclusion, toxin-antitoxin systems are ubiquitous in both bacterial chromosomes and plasmids. So far, most researches have focused on Gram-negative bacteria, especially in E. coli; in contrast, information on Gram-positive bacteria is quite limited. Discoveries include the relBE and mazEF systems in Streptococcus mutans and enterococci [34, 35], the talAB system in Leifsonia xyli [36], the Rv1102cRv1103c system in Mycobacterium tuberculosis [37], and so forth. Further examples are shown in Tables 1 and 2. These TA systems are suggested to have similar functions to their homologues in Gram-negative bacteria.

\section{3. mazEF System and Bacterial PCD}

As the best studied bacterial PCD module, mazEF system has the following features: (i) MazF is toxic and MazE is antitoxic [38]. MazF is a sequence-specific endoribonuclease, which cleaves mRNA between $\mathrm{A}$ and $\mathrm{C}$ at the ACA sequence [39, 40]; (ii) MazF is long-lived, whereas MazE is a labile protein degraded in vivo by the ATP-dependent ClpPA serine protease [41]; (iii) MazE and MazF have the ability to associate into $\mathrm{MazF}_{2}-\mathrm{MazE}_{2}-\mathrm{MazF}_{2}$ heterohexamers in physical conditions [42]; (iv) MazE and MazF are coexpressed, located downstream the relA gene in the rel operon [41]; (v) mazEF is negatively autoregulated at the transcription level by the combined action of both MazE and MazF proteins [43].

Several investigations have revealed that some stress conditions, including starvation, antibiotics, high temperature, DNA damage, and oxidative stress, can trigger bacterial cell death through mazEF system $[24,44,45]$. Guanosine $3^{\prime}, 5^{\prime}$ bispyrophosphate (ppGpp) has been revealed to regulate the starvation-triggered PCD [41]. As the key signal molecule signs of amino acids deficiency, the synthesis of ppGpp 
TABLE 1: Properties of well-characterized TA systems in plasmids.

\begin{tabular}{|c|c|c|c|c|c|c|c|}
\hline \multirow{2}{*}{ TA system } & \multirow{2}{*}{ Organism } & \multirow{2}{*}{ Toxin } & \multicolumn{2}{|c|}{ Antitoxin } & \multirow{2}{*}{ Target } & \multirow{2}{*}{ Protease cleaving the antitoxin } & \multirow{2}{*}{ Reference } \\
\hline & & & Protein & RNA & & & \\
\hline$c c d A B$ & E. coli & $\mathrm{CcdB}$ & CcdA & & DNA gyrase & Lon & {$[46,47]$} \\
\hline $\operatorname{din} J-y a f Q$ & E. coli & YafQ & DinJ & & Unknown & Unknown & {$[48]$} \\
\hline hok/sok & E. coli & Sok & & Hok & Cell membrane & Unknown & {$[49,50]$} \\
\hline kid/kis & E. coli & Kid & Kis & & mRNA cleavage & Lon & {$[51]$} \\
\hline parDE & E. coli & ParE & ParD & & DNA gyrase & Unknown & {$[52]$} \\
\hline pemKI & E. coli & PemK & PemI & & DnaB & Lon & {$[53]$} \\
\hline relBE & E. coli & RelE & RelB & & mRNA cleavage & Unknown & {$[16]$} \\
\hline yefM-yoeB & E. coli & YoeB & YefM & & mRNA cleavage & Unknown & {$[54]$} \\
\hline relBE2 & S. pneumoniae & RelE & RelB & & Unknown & Unknown & {$[28]$} \\
\hline mazEF & enterococci & MazF & MazE & & mRNA cleavage & ClpPA & {$[34]$} \\
\hline higBA & $\begin{array}{l}\text { V. cholerae } \\
\text { P. vulgaris }\end{array}$ & HigB & HigA & & mRNA cleavage & Unknown & {$[55,56]$} \\
\hline phd-doc & V. cholerae & Phd & Doc & & $30 \mathrm{~S}$ ribosomal subunit & ClpXP & {$[57]$} \\
\hline toxIN & E. carotovora & ToxN & & ToxI & Unknown & Unknown & {$[7]$} \\
\hline axe-txe & E. faecium & Txe & Axe & & Unknown & Unknown & [58] \\
\hline$\omega-\varepsilon-\zeta$ & $\begin{array}{l}\text { E. faecium } \\
\text { S. pyogenes }\end{array}$ & $\zeta$ & $\varepsilon$ & & Transcription & Unknown & {$[59,60]$} \\
\hline fst & $\begin{array}{l}\text { E. faecalis } \\
\text { B. subtilis }\end{array}$ & Fst(RNA I) & & RNA II & Translation & Unknown & {$[61,62]$} \\
\hline mvpTA & Sh. flexneri & MvpT & MvpA & & Unknown & Unknown & {$[63]$} \\
\hline
\end{tabular}

TABLE 2: Properties of TA systems in chromosomes.

\begin{tabular}{|c|c|c|c|c|c|c|c|}
\hline \multirow{2}{*}{ TA system } & \multirow{2}{*}{ Organism } & \multirow{2}{*}{ Toxin } & \multicolumn{2}{|c|}{ Antitoxin } & \multirow{2}{*}{ Target } & \multirow{2}{*}{ Protease cleaving the antitoxin } & \multirow{2}{*}{ Reference } \\
\hline & & & Protein & Antisense RNA & & & \\
\hline$\overline{c c d A B}$ & E. coli & $\mathrm{CcdB}$ & CcdA & & DNA gyrase & Lon & {$[64]$} \\
\hline $\operatorname{chpBIK}$ & E. coli & ChpBK & ChpBI & & mRNA cleavage & Unknown & {$[25]$} \\
\hline dinJ-yafQ & E. coli & YafQ & DinJ & & mRNA cleavage & Unknown & {$[30,65]$} \\
\hline hipBA & E. coli & HipA & НipВ & & Unknown & Unknown & {$[66]$} \\
\hline mazEF & E. coli & $\mathrm{MazF}$ & MazE & & mRNA cleavage & ClpPA & [23] \\
\hline prlF-yhaV & E. coli & YhaV & $\operatorname{PrlF}$ & & Unknown & Unknown & {$[31]$} \\
\hline relBE & E. coli & RelE & RelB & & mRNA cleavage & Lon & [67] \\
\hline istR-tisB & E. coli & TisB & & IstR-1 & Cell membrane & Unknown & {$[68]$} \\
\hline$m q s A-m q s R$ & E. coli & MqsR & MqsA & & RNA cleavage & Lon, ClpXP & [69] \\
\hline hicBA & E. coli & HicA & $\mathrm{HicB}$ & & mRNA cleavage & Lon & [70] \\
\hline yaf $N-y a f O$ & E. coli & YafO & YafN & & mRNA cleavage & Unknown & [71] \\
\hline yefM-yoeB & E. coli, S. pneumoniae & YoeB & YefM & & mRNA cleavage & Unknown & {$[54]$} \\
\hline relBE2 & S. pneumoniae & RelE & RelB & & Unknown & Unknown & {$[28]$} \\
\hline pezTA $(\varepsilon-\zeta$ like $)$ & S. pneumoniae & PezT & PezA & & Transcription & Unknown & {$[72]$} \\
\hline$y d c D E$ & B. subtilis & YdcE & YdcD & & Translation? & Unknown & [73] \\
\hline pemKI & B. anthracis & PemK & PemI & & DnaB & Lon & {$[74]$} \\
\hline phd-doc & N. europaea & Phd & Doc & & Translation & ClpXP & {$[75]$} \\
\hline parDE & N. europaea & ParE & ParD & & DNA gyrase & Unknown & [75] \\
\hline rv1991ac & M. tuberculosis & Rv1991a & Rv1991c & & Unknown & Unknown & {$[76]$} \\
\hline $\operatorname{vap} B C$ & M. smegmatis & VapC & VapB & & mRNA cleavage & Unknown & {$[77,78]$} \\
\hline higBA & V. cholerae & HigB & HigA & & Unknown & Unknown & [79] \\
\hline mosA-mosT & V. cholerae & MosT & MosA & & Unknown & Unknown & [80] \\
\hline$r v 1102 c-1103 c$ & M. tuberculosis & Rv1102c & Rv1103c & & mRNA cleavage & Unknown & [37] \\
\hline talAB & L. xyli & TalB & TalA & & Unknown & Unknown & {$[36]$} \\
\hline
\end{tabular}


in E. coli is governed by two pathways. One is activated by amino acid deprivation. The enzyme responsible for this pathway is encoded by relA, which is induced by uncharged tRNA or inhibited by amino acylation. The other pathway is activated by the limitation of carbon source, which is relA-independent [81]. It is indicated that under nutritional starvation conditions, increased ppGpp will then inhibit the coexpression of mazE and mazF. As MazE is decreased rapidly, MazF can exert its toxic effect and cause cell death. Artificial overproduction of ppGpp could by itself cause mazEF-dependent cell death, providing an experimental evidence for the model above [41].

Bacterial PCD mediated by mazEF is also a population phenomenon. Antibiotics inhibiting transcription and/or translation (such as rifampicin and chloramphenicol) can prevent the synthesis of the short-lived antitoxin MazE and then induce PCD in E. coli [44]. This interesting finding leads to the discovery of a novel linear pentapeptide quorum sensing signal (an extracellular death factor, EDF): AsnAsn-Trp-Asn-Asn (NNWNN) [82]. This signal peptide is essential for mazEF-mediated cell death [83], and thus necessary for reactive oxygen species (ROS) production [84]. Previous studies have confirmed that antibiotics inhibiting transcription and/or translation can cause mazEFmediated PCD by forming ROS. Things are different for other antibiotics that cause DNA damage (like nalidixic acid); they also induce ROS production, however, arouse mazEF-mediated PCD through an ROS-independent way [85]. Further investigation by Amitai et al. discovered several "survival proteins" that are only triggered in the ROS-dependent pathway [86]. This provides a perspective in understanding how bacteria deal with different stress conditions and how to choose between living and death.

Mechanisms involved in mazEF-mediated PCD are briefly summarized in Figure 1. It is clear to see that EDF and ROS are the crux of the matter. Kolodkin-Gal et al. [82] searched the entire E. coli genome for DNA sequences encoding the amino acid sequence of NNWNN and found that EDF most likely comes from the modification of glucose-6-phosphate dehydrogenase (G6PD). G6PD is a key metabolic enzyme to catalyze the first and rate-limiting step of the pentose phosphate shunting and produce nucleotide precursors [87]. Even more interestingly, as oxidative stress is the major death inducer in both prokaryotes and eukaryotes, the role of G6PD in diminishing oxidative stress and protecting mammalian cells from apoptosis has been reported recently, and ROS modulation is essential for cytosolic cytochrome $\mathrm{C}$ to fully activate caspases and apoptosis [88]. Therefore, it may be reasonable to infer that G6PD might also play an important role in bacterial PCD regulation, and that EDF generated from G6PD may link metabolism and PCD through ROS.

\section{PCD Related to Bacterial Biofilm}

As structured multicellular communities, biofilms can be found in diverse environments. Biofilm strategy is adopted to protect bacteria from many environmental stresses (e.g., antibiotics, UV lights, or heavy metal toxicities), realize better use of nutrient, acquire metabolic cooperativity, and even obtain new genetic traits [89]. Researchers have demonstrated that cells at different zones of biofilm show different metabolic activities, with those embedded in the center having the lowest activity $[90,91]$. Many recent studies have suggested that bacterial PCD played a significant role in biofilm formation and development $[92,93]$. In such a structure, PCD may have multiple functions like (i) creating water channels within biofilms to transport nutrients or waste into and from cells deeply inside; (ii) releasing extracellular DNA that can be used for structuring biofilms [94]; and (iii) allowing cells to dispersal from the biofilm matrix when bacteria need to escape the architecture [95].

Although cell death and lysis are definitely linked to biofilm formation $[92,93,96]$, reports on the effect of TA systems on biofilm formation are limited. Tsilibaris et al. constructed a mutant $E$. coli strain MG1655 that lacks the five best-studied proteic TA systems ( $\Delta 5, \mathrm{MazF} / \mathrm{MazE}$, RelE/RelB, ChpB, YoeB/YefM, and YafQ/DinJ) and found no significant difference between wild type and $\Delta 5$ under various PCDinducing conditions [97]. This result raised a big question on the real function of TA system located on chromosomes. Using these two strains and E. coli BW25113, Kim et al. investigated whether these five TA loci affect biofilm formation. They found that compared to MG1655 WT, $\Delta 5$ decreased biofilm formation at $8 \mathrm{~h}$ and increased biofilm formation at $24 \mathrm{~h}$. This result is caused by YjgK overexpression, a protein affecting the production of fimbriae involved in both biofilm attachment and dispersal. Furthermore, a toxin Hha and an antitoxin YefM may be involved in biofilm formation. In fact, deletions of each of the five toxins and overexpressions in another genetic background strain BW25113 have totally opposite results in biofilm formation [95]. Then, it is interesting to notice that as Kolodkin-Gal and EngelbergKulka described recently, unlike many well-studied E. coli strains, strain MG1655 used in the experiments mentioned above is partially defective both in production and response to EDF; thus mazEF-mediated cell death does not occur [83]. Following this result, they further studied how each of these five TA systems affected bacterial cell death differently during biofilm formation in E. coli strain MC4100 [98]. Their findings are as follows: (i) mazEF is the regulating module mediating cell death both in liquid media and in biofilm formation; (ii) relBE seems to be a principal mediator of cell death only in liquid media, but not in biofilm formation; (iii) $c h p B I K$ seems to be a back-up death system for mazEF in a ppGpp-independent way; (iv) yefM-yoeB mediates cell death only in liquid media at some conditions, while not at all in biofilm formation; ( $\mathrm{v}$ ) dinJ-yafQ seems to be a principal mediator of cell death only in biofilm formation. Compared with previous studies on MG1655 that can be seen as phenotypically $\mathrm{MazEF}^{-}$, it is tempting to infer that many TA systems might connect each other as a network where mazEF plays as a kind of effector to activate death path under some conditions, while some other TA loci may form alternative regulatory pathways to deal with other stresses. 


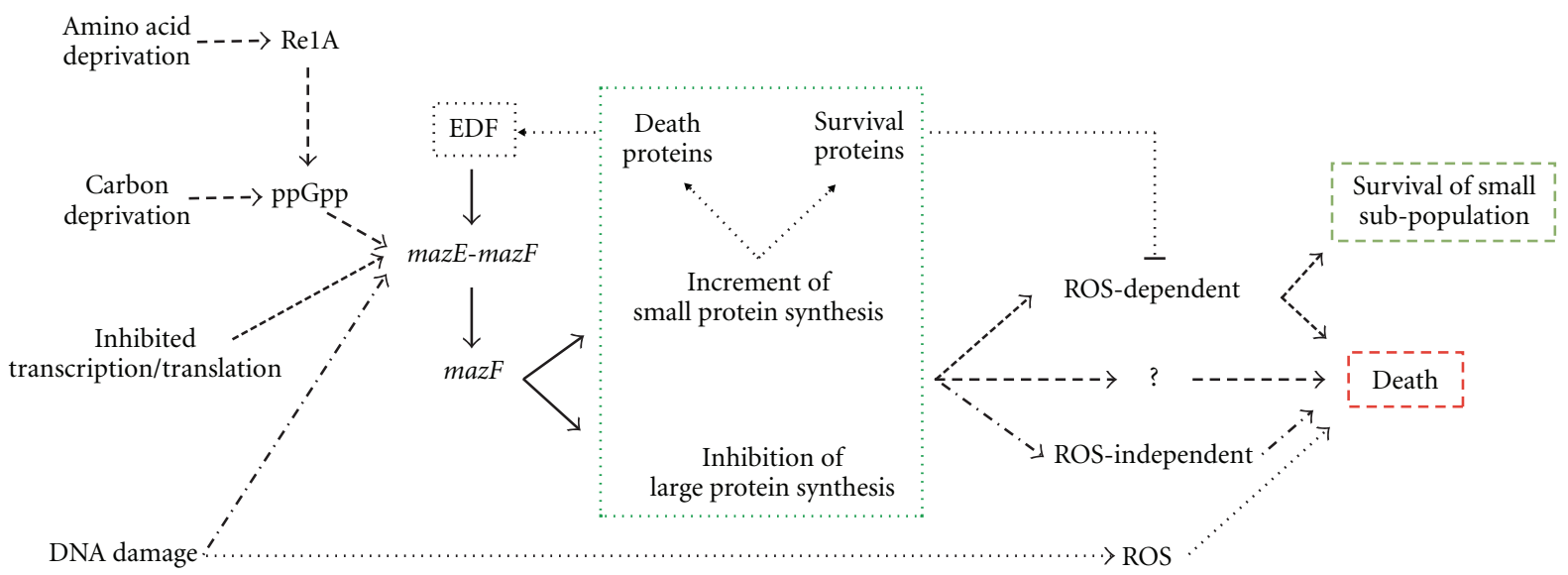

$--\rightarrow$ Starvation routs mediated by ppGpp
$--\rightarrow$ Antibiotics which inhibit transcription/translation
$-\rightarrow$ Antibiotics that cause DNA damage
$\ldots . \rightarrow$ Other PCD pathways triggered by DNA damage
$\rightarrow$ Common mechanisms exist in all stress conditions mentioned above
$\ldots . . \rightarrow$ Regulation pathways involved in mazEF-mediated bacterial PCD

Figure 1: Mechanisms involved in mazEF-mediated bacterial PCD. mazEF-mediated bacterial PCD can be triggered by several stress conditions including starvation, antibiotics, DNA damage, and so forth.

This speculation feels more possible when the studies go further to the TA systems connected with persister cell formation, which is a small fraction of bacteria that resist to antibiotics without genetic change forming during the stationary-phase or in biofilm. Kim et al. found that MqsR and MqsA of E. coli are toxin-antitoxin pair that influences cell physiology (e.g., biofilm formation and motility) via RNase activity as well as through regulation of toxin CspD [69]. They identified eight genes ( $\operatorname{csp} D, \operatorname{clpX}$, clpP, lon, $y f j Z$, relB, relE, and hokA) related to MqsR toxicity and discovered that toxins CspD, Hha, and HokA influence persister cell formation via MqsR and small RNA regulator Hfq [99]. Besides, it is interesting to notice that (i) ClpXP is an important protease system for stress-induced environments and degrades RpoS and Dps; (ii) many antitoxins, including MazE and RelB, are degraded by the ATP-dependent ClpP and Lon proteases (Tables 1 and 2); (iii) Besides normally taking place during exponential growth, mazEF-mediated cell death also occurs in a $\triangle r p o S$ mutant at stationary phase, which means RpoS is responsible for the resistance to mazEFmediated PCD during stationary growth [100]; (iv) hha is induced dramatically in biofilm. According to the studies on MG1655 and $\Delta 5$, the ability of Hha to reduce biofilm formation is dependent on the activity of some of these five TA systems [95]. Furthermore, MqsR is involved in the regulation of motility signaled by quorum sensing factor autoinducer-2 (AI-2). Based on results mentioned above, it is meaningful to investigate TA systems from an interactive view.

\section{The Possible Roles of Chromosomal TA Systems}

Inspecting entire history of biological evolution, although it is filled with competitions among individuals and species, it is undeniable that collaborations still exist. In particular, some altruistic behaviors like PCD were found in unicellular organism (such as bacteria) in recent years. As we mainly discussed above, a group of researchers suggested that at least some of the TA systems act as apoptotic tools. Bacteria can obtain a lot of benefit from this function, as Engelberg-Kulka et al. have pointed out there are three possible functions for PCD systems [101]. Firstly, under nutritional starvation conditions, the death of a part of bacterial individuals can provide food for the survivals to maintain the species. Secondly, PCD acts as a defense approach to prevent the spreading of phage infection, for example, PCD mediated by mazEF can prevent the spreading of phage P1 [23]. Thirdly, PCD can also act as a guardian of the bacterial chromosome. When other systems fail, PCD systems might maintain genomic stability by elimination of deficient cells and/or mutations from the culture. The so-called "PCD hypothesis" is represented primarily by mazEF module.

However, question marks are still raised on the functions of chromosomal toxin-antitoxin systems. As an alternative hypothesis, another group of scientists $[49,102]$ suggested that rather than killing the cell, TA systems let the bacteria enter a latent state from which they can recover under favorable conditions and function as metabolic regulators. 
Take relBE for an example: RelE is a kind of mRNA interferases that must cleave mRNA at the ribosomal A site, which is translation-dependent. When bacteria facing nutritional deprivation or other stressful conditions, RelE becomes active and reduces the global rate of translation by mRNA cleavage, causing the cell growth arrest [67], and when the "difficult time" has passed, newly synthesized RelB neutralizes the toxin, letting the cell return to the normal life [103]. First suggested by Pedersen et al. [102], this hypothesis was then challenged by Amitai et al. [38] showing the existence of a no return point in MazF lethality that occurs sooner in minimal growth medium than in rich medium. The point of no return in the action of MazF was further confirmed by Kolodkin-Gal and Engelberg-Kulka [104]. However, many other TA systems, including relBE, are still proved to be bacteriostasis. More information has been provided in several excellent reviews [105-107].

Besides, some authors believe that these two different assumptions are not completely incompatible. They suggested that TA systems may be activated by stress and bring cells into stasis. However, escaping from that state may be different in various cells: some of the population may die in the course of transition, while some "lucky beggars" may obtain nutrition at the expense of their less lucky neighbors [107].

The existence of problems and controversies reflects the complexity of the TA systems and their versatility of the functions. Recently, at least nine possible functions in chromosome TA systems have been summarized, including junk, stabilization of genomic parasites, selfish alleles, gene regulation, growth control, persisters [108], programmed cell arrest and the preservation of the commons, programmed cell death, antiphage, and so forth $[109,110]$. Each chromosome TA system has been evaluated to take part in at least one of the functions. These functions are undoubtedly significant to bacterial collaborative behaviors. The death of a subpopulation may enable to provide construction matrix components (such as the extracellular genomic DNA) for others to form biofilm or persister cell, or to give up some nutrients for others to generate a metabolic activity. This may again draw attention on the speculation we mentioned above: each TA system may deal with different problems through different aspects, and they collaborate with each other, form an exquisite network, to help the species survive.

\section{Perspectives}

The process of bacterial PCD is an interesting problem, which on the first sight seemed to be a paradoxical phenomenon as the final purpose is survival not death. At present, it is far from clear on this complex physiological process. The remaining questions are how bacteria make the most beneficial choice between living and death, what pivotal death factors in different bacterial groups are, what happens under the direction of the death signals in this process, especially when biofilm formation is involved.

Another question is that although both bacterial PCD and apoptosis are deeply explored, whether they have evolutionary connection is not clear enough. In fact, by comparing the apoptosis with the bacterial PCD, a significant similarity is found between them [111]. For example, the mechanism of toxin/antitoxin mentioned above is similar to the IAPs/caspase (IAPs is a family of proteins that can inhibit caspase function by binding of their IAP repeat domain to the caspase active site, promoting the degradation of active caspases, or sequestering the caspase away from its substrates) in Drosophila melanogaster [112]. This similarity might suggest a common ancestor of those systems, providing us some novel perspectives for the further study.

Furthermore, the potential value of bacterial PCD in disease control is great. Bacterial PCD provides us an absolute new idea in designing more efficient "antibiotics" $[84,85,101]$, which can activate bacterial suicide modules and cause bacterial death in a more special manner. This kind of achievement surely will produce far-reaching effects on human health and present an attractive researching field for the modern biochemists.

\section{Acknowledgment}

This work was supported by the National Natural Science Foundation of China Grant no. 30870055 (to Y.-J. Feng). M.$\mathrm{x}$. Hu, X. Zhang, and E.-l. Li contributed equally to this work.

\section{References}

[1] H. Engelberg-Kulka, S. Amitai, I. Kolodkin-Gal, and R. Hazan, "Bacterial programmed cell death and multicellular behavior in bacteria," PLoS Genetics, vol. 2, no. 10, pp. 1518 1526, 2006.

[2] M. D. Jacobson, M. Weil, and M. C. Raff, "Programmed cell death in animal development," Cell, vol. 88, no. 3, pp. 347354, 1997.

[3] S. K. Christensen, G. Maenhaut-Michel, N. Mine, S. Gottesman, K. Gerdes, and L. Van Melderen, "Overproduction of the Lon protease triggers inhibition of translation in Escherichia coli: involvement of the yefM-yoeB toxinantitoxin system," Molecular Microbiology, vol. 51, no. 6, pp. 1705-1717, 2004.

[4] R. B. Jensen and K. Gerdes, "Programmed cell death in bacteria: proteic plasmid stabilization systems," Molecular Microbiology, vol. 17, no. 2, pp. 205-210, 1995.

[5] E. M. Fozo, M. R. Hemm, and G. Storz, "Small toxic proteins and the antisense RNAs that repress them," Microbiology and Molecular Biology Reviews, vol. 72, no. 4, pp. 579-589, 2008.

[6] K. S. Makarova, Y. I. Wolf, and E. V. Koonin, "Comprehensive comparative-genomic analysis of type 2 toxinantitoxin systems and related mobile stress response systems in prokaryotes," Biology Direct, vol. 4, article 19, 2009.

[7] P. C. Fineran, T. R. Blower, I. J. Foulds, D. P. Humphreys, K. S. Lilley, and G. P. C. Salmond, "The phage abortive infection system, ToxIN, functions as a protein-RNA toxin-antitoxin pair," Proceedings of the National Academy of Sciences of the United States of America, vol. 106, no. 3, pp. 894-899, 2009.

[8] E. W. Sevin and F. Barloy-Hubler, "RASTA-Bacteria: a webbased tool for identifying toxin-antitoxin loci in prokaryotes," Genome Biology, vol. 8, no. 8, article R155, 2007.

[9] E. M. Fozo, K. S. Makarova, S. A. Shabalina, N. Yutin, E. V. Koonin, and G. Storz, "Abundance of type I toxinantitoxin systems in bacteria: searches for new candidates and 
discovery of novel families," Nucleic Acids Research, vol. 38, no. 11, pp. 3743-3759, 2010.

[10] Y. Masuda, K. Miyakawa, Y. Nishimura, and E. Ohtsubo, "chpA and $\operatorname{chpB}$, Escherichia coli chromosomal homologs of the pem locus responsible for stable maintenance of plasmid R100," Journal of Bacteriology, vol. 175, no. 21, pp. 68506856, 1993.

[11] T. Ogura and S. Hiraga, "Mini-F plasmid genes that couple host cell division to plasmid proliferation," Proceedings of the National Academy of Sciences of the United States of America, vol. 80, no. 15, pp. 4784-4788, 1983.

[12] R. Loris, M. H. Dao-Thi, E. M. Bahassi et al., "Crystal structure of $\mathrm{CcdB}$, a topoisomerase poison from E. coli," Journal of Molecular Biology, vol. 285, no. 4, pp. 1667-1677, 1999.

[13] L. Van Melderen, M. H. D. Thi, P. Lecchi, S. Gottesman, M. Couturier, and M. R. Maurizi, "ATP-dependent degradation of CcdA by Lon protease. Effects of secondary structure and heterologous subunit interactions," Journal of Biological Chemistry, vol. 271, no. 44, pp. 27730-27738, 1996.

[14] T. Madl, L. Van Melderen, N. Mine et al., "Structural basis for nucleic acid and toxin recognition of the bacterial antitoxin CcdA," Journal of Molecular Biology, vol. 364, no. 2, pp. 170$185,2006$.

[15] P. Bernard and M. Couturier, "Cell killing by the F plasmid CcdB protein involves poisoning of DNA-topoisomerase II complexes," Journal of Molecular Biology, vol. 226, no. 3, pp. 735-745, 1992.

[16] H. Grønlund and K. Gerdes, "Toxin-antitoxin systems homologous with relBE of Escherichia coli plasmid P307 are ubiquitous in prokaryotes," Journal of Molecular Biology, vol. 285, no. 4, pp. 1401-1415, 1999.

[17] A. J. Muñoz-Gómez, M. Lemonnier, S. Santos-Sierra, A. Berzal-Herranz, and R. Díaz-Orejas, "RNase/anti-RNase activities of the bacterial parD toxin-antitoxin system," Journal of Bacteriology, vol. 187, no. 9, pp. 3151-3157, 2005.

[18] S. Santos-Sierra, M. Lemonnier, B. Nuñez et al., "Noncytotoxic variants of the Kid protein that retain their autoregulatory activity," Plasmid, vol. 50, no. 2, pp. 120-130, 2003.

[19] D. Hargreaves, S. Santos-Sierra, R. Giraldo et al., "Structural and functional analysis of the kid toxin protein from E. coli plasmid R1," Structure, vol. 10, no. 10, pp. 1425-1433, 2002.

[20] S. Santos-Sierra, C. Pardo-Abarrio, R. Giraldo, and R. DíazOrejas, "Genetic identification of two functional regions in the antitoxin of the parD killer system of plasmid R1," FEMS Microbiology Letters, vol. 206, no. 1, pp. 115-119, 2002.

[21] F. Hayes, "Toxins-antitoxins: plasmid maintenance, programmed cell death, and cell cycle arrest," Science, vol. 301, no. 5639, pp. 1496-1499, 2003.

[22] C. S. Hayes and R. T. Sauer, "Toxin-antitoxin pairs in bacteria: killers or stress regulators?" Cell, vol. 112, no. 1, pp. 2-4, 2003.

[23] R. Hazan and H. Engelberg-Kulka, "Escherichia coli mazEFmediated cell death as a defense mechanism that inhibits the spread of phage P1," Molecular Genetics and Genomics, vol. 272, no. 2, pp. 227-234, 2004.

[24] R. Hazan, B. Sat, and H. Engelberg-Kulka, "Escherichia coli mazEF-mediated cell death is triggered by various stressful conditions," Journal of Bacteriology, vol. 186, no. 11, pp. 3663-3669, 2004.
[25] Y. Zhang, L. Zhu, J. Zhang, and M. Inouye, "Characterization of ChpBK, an mRNA interferase from Escherichia coli," Journal of Biological Chemistry, vol. 280, no. 28, pp. 2608026088, 2005.

[26] M. Gotfredsen and K. Gerdes, "The Escherichia coli relBE genes belong to a new toxin-antitoxin gene family," Molecular Microbiology, vol. 29, no. 4, pp. 1065-1076, 1998.

[27] G. Y. Li, Y. Zhang, M. Inouye, and M. Ikura, "Structural mechanism of transcriptional autorepression of the Escherichia coli RelB/RelE antitoxin/toxin module," Journal of Molecular Biology, vol. 380, no. 1, pp. 107-119, 2008.

[28] C. Nieto, T. Pellicer, D. Balsa, S. K. Christensen, K. Gerdes, and M. Espinosa, "The chromosomal relBE2 toxin-antitoxin locus of Streptococcus pneumoniae: characterization and use of a bioluminescence resonance energy transfer assay to detect toxin-antitoxin interaction," Molecular Microbiology, vol. 59, no. 4, pp. 1280-1296, 2006.

[29] S. E. S. Bailey and F. Hayes, "Influence of operator site geometry on transcriptional control by the yefM-yoeB toxinantitoxin complex," Journal of Bacteriology, vol. 191, no. 3, pp. 762-772, 2009.

[30] R. Motiejûnaitë, J. Armalytë, A. Markuckas, and E. Sužiedeliene, "Escherichia coli dinJ-yafQ genes act as a toxinantitoxin module," FEMS Microbiology Letters, vol. 268, no. 1, pp. 112-119, 2007.

[31] O. Schmidt, V. J. Schuenemann, N. J. Hand et al., "prlF and yhaV encode a new toxin-antitoxin system in Escherichia coli," Journal of Molecular Biology, vol. 372, no. 4, pp. 894905, 2007.

[32] S. B. Korch, H. Contreras, and J. E. Clark-Curtiss, "Three Mycobacterium tuberculosis rel toxin-antitoxin modules inhibit mycobacterial growth and are expressed in infected human macrophages," Journal of Bacteriology, vol. 191, no. 5, pp. 1618-1630, 2009.

[33] H. Nariya and M. Inouye, "MazF, an mRNA interferase, mediates programmed cell death during multicellular Myxococcus development," Cell, vol. 132, no. 1, pp. 55-66, 2008.

[34] E. M. Moritz and P. J. Hergenrother, "Toxin-antitoxin systems are ubiquitous and plasmid-encoded in vancomycinresistant enterococci," Proceedings of the National Academy of Sciences of the United States of America, vol. 104, no. 1, pp. 311-316, 2006.

[35] J. A. C. Lemos, T. A. Brown Jr., J. Abranches, and R. A. Burne, "Characteristics of Streptococcus mutans strains lacking the mazEF and RelBE toxin-antitoxin modules," FEMS Microbiology Letters, vol. 253, no. 2, pp. 251-257, 2005.

[36] L. Cheng, H. Lin, X. Fan et al., "A novel toxin-antitoxin operon talA/B from the Gram-positive bacterium Leifsonia xyli subsp. cynodontis," FEBS Letters, vol. 582, no. 21-22, pp. 3211-3216, 2008.

[37] J.-S. Han, J. J. Lee, T. Anandan et al., "Characterization of a chromosomal toxin-antitoxin, Rv1102c-Rv1103c system in Mycobacterium tuberculosis," Biochemical and Biophysical Research Communications, vol. 400, no. 3, pp. 293-298, 2010.

[38] S. Amitai, Y. Yassin, and H. Engelberg-Kulka, "MazFmediated cell death in Escherichia coli: a point of no return," Journal of Bacteriology, vol. 186, no. 24, pp. 8295-8300, 2004.

[39] Y. Zhang, J. Zhang, K. P. Hoeflich, M. Ikura, G. Qing, and M. Inouye, "MazF cleaves cellular mRNAs specifically at ACA to block protein synthesis in Escherichia coli," Molecular Cell, vol. 12, no. 4, pp. 913-923, 2003. 
[40] Y. Zhang, J. Zhang, H. Hara, I. Kato, and M. Inouye, "Insights into the mRNA cleavage mechanism by MazF, an mRNA interferase," Journal of Biological Chemistry, vol. 280, no. 5, pp. 3143-3150, 2004.

[41] E. Aizenman, H. Engelberg-Kulka, and G. Glaser, "An Escherichia coli chromosomal "addiction module" regulated by $3^{\prime}, 5^{\prime}$-bispyrophosphate: a model for programmed bacterial cell death," Proceedings of the National Academy of Sciences of the United States of America, vol. 93, no. 12, pp. 6059-6063, 1996.

[42] K. Kamada, F. Hanaoka, and S. K. Burley, "Crystal structure of the MazE/MazF complex: molecular bases of antidotetoxin recognition," Molecular Cell, vol. 11, no. 4, pp. 875-884, 2003.

[43] I. Marianovsky, E. Aizenman, H. Engelberg-Kulka, and G. Glaser, "The regulation of the Escherichia coli mazEF promoter involves an unusual alternating palindrome," Journal of Biological Chemistry, vol. 276, no. 8, pp. 5975-5984, 2001.

[44] B. Sat, R. Hazan, T. Fisher, H. Khaner, G. Glaser, and H. Engelberg-Kulka, "Programmed cell death in Escherichia coli: some antibiotics can trigger mazEF lethality," Journal of Bacteriology, vol. 183, no. 6, pp. 2041-2045, 2001.

[45] B. Sat, M. Reches, and H. Engelberg-Kulka, "The Escherichia coli chromosomal suicidal module mazEF is involved in thymine-less death," Journal of Bacteriology, vol. 185, no. 6, pp. 1803-1807, 2003.

[46] A. Jaffe, T. Ogura, and S. Hiraga, "Effects of the $c c d$ function of the F plasmid on bacterial growth," Journal of Bacteriology, vol. 163, no. 3, pp. 841-849, 1985.

[47] M. H. Dao-Thi, D. Charlier, R. Loris et al., "Intricate interactions within the $c c d$ plasmid addiction system," Journal of Biological Chemistry, vol. 277, no. 5, pp. 3733-3742, 2002.

[48] R. Motiejūnaite, J. Armalytè, V. Šeputienè, and E. Sužiedèlienè, "Escherichia coli dinJ-yafQ operon shows characteristic features of bacterial toxin-antitoxin modules," Biologija, vol. 4, pp. 9-14, 2005.

[49] T. Franch and K. Gerdes, "Programmed cell death in bacteria: translational repression by mRNA end-pairing," Molecular Microbiology, vol. 21, no. 5, pp. 1049-1060, 1996.

[50] K. Wu and T. K. Wood, "Evaluation of the hok/sok killer locus for enhanced plasmid stability," Biotechnology and Bioengineering, vol. 44, no. 8, pp. 912-921, 1994.

[51] B. Pimentel, M. A. Madine, and G. De La Cueva-Méndez, "Kid cleaves specific mRNAs at UUACU sites to rescue the copy number of plasmid R1," EMBO Journal, vol. 24, no. 19, pp. 3459-3469, 2005.

[52] A. Bravo, G. de Torrontegui, and R. Díaz, "Identification of components of a new stability system of plasmid R1, parD, that is close to the origin of replication of this plasmid," $M G G$ Molecular \& General Genetics, vol. 210, no. 1, pp. 101-110, 1987.

[53] J. Zhang, Y. Zhang, L. Zhu, M. Suzuki, and M. Inouye, "Interference of mRNA function by sequence-specific endoribonuclease PemK," Journal of Biological Chemistry, vol. 279, no. 20, pp. 20678-20684, 2004.

[54] C. Nieto, I. Cherny, K. K. Seok et al., "The yefM-yoeB toxin-antitoxin systems of Escherichia coli and Streptococcus pneumoniae: functional and structural correlation," Journal of Bacteriology, vol. 189, no. 4, pp. 1266-1278, 2007.

[55] M. Christensen-Dalsgaard and K. Gerdes, "Two higBA loci in the Vibrio cholerae superintegron encode mRNA cleaving enzymes and can stabilize plasmids," Molecular Microbiology, vol. 62, no. 2, pp. 397-411, 2006.
[56] J. M. Hurley and N. A. Woychik, "Bacterial toxin HigB associates with ribosomes and mediates translation-dependent mRNA cleavage at A-rich sites," Journal of Biological Chemistry, vol. 284, no. 28, pp. 18605-18613, 2009.

[57] M. Liu, Y. Zhang, M. Inouye, and N. A. Woychik, "Bacterial addiction module toxin Doc inhibits translation elongation through its association with the 30 S ribosomal subunit," Proceedings of the National Academy of Sciences of the United States of America, vol. 105, no. 15, pp. 5885-5890, 2008.

[58] R. Grady and F. Hayes, "Axe-Txe, a broad-spectrum proteic toxin-antitoxin system specified by a multidrug-resistant, clinical isolate of Enterococcus faecium," Molecular Microbiology, vol. 47, no. 5, pp. 1419-1432, 2003.

[59] H. Sletvold, P. J. Johnsen, I. Hamre, G. S. Simonsen, A. Sundsfjord, and K. M. Nielsen, "Complete sequence of Enterococcus faecium pVEF3 and the detection of an $\omega-\varepsilon-\zeta$ toxin-antitoxin module and an ABC transporter," Plasmid, vol. 60, no. 1, pp. 75-85, 2008.

[60] U. Zielenkiewicz, M. Kowalewska, C. Kaczor, and P. Cegłowski, "In vivo interactions between toxin-antitoxin proteins epsilon and zeta of streptococcal plasmid pSM19035 in Saccharomyces cerevisiae," Journal of Bacteriology, vol. 191, no. 11, pp. 3677-3684, 2009.

[61] S. Shokeen, S. Patel, T. J. Greenfield, C. Brinkman, and K. E. Weaver, "Translational regulation by an intramolecular stemloop is required for intermolecular RNA regulation of the par addiction module," Journal of Bacteriology, vol. 190, no. 18, pp. 6076-6083, 2008.

[62] S. Patel and K. E. Weaver, "Addiction toxin Fst has unique effects on chromosome segregation and cell division in Enterococcus faecalis and Bacillus subtilis," Journal of Bacteriology, vol. 188, no. 15, pp. 5374-5384, 2006.

[63] S. Sayeed, L. Reaves, L. Radnedge, and S. Austin, "The stability region of the large virulence plasmid of Shigella flexneri encodes an efficient postsegregational killing system," Journal of Bacteriology, vol. 182, no. 9, pp. 2416-2421, 2000.

[64] M. Wilbaux, N. Mine, A. M. Guérout, D. Mazel, and L. Van Melderen, "Functional interactions between coexisting toxin-antitoxin systems of the $c c d$ family in Escherichia coli O157:H7," Journal of Bacteriology, vol. 189, no. 7, pp. 27122719, 2007.

[65] M. H. Prysak, C. J. Mozdzierz, A. M. Cook et al., "Bacterial toxin YafQ is an endoribonuclease that associates with the ribosome and blocks translation elongation through sequence-specific and frame-dependent mRNA cleavage," Molecular Microbiology, vol. 71, no. 5, pp. 1071-1087, 2009.

[66] F. F. Correia, A. D’Onofrio, T. Rejtar et al., "Kinase activity of overexpressed HipA is required for growth arrest and multidrug tolerance in Escherichia coli," Journal of Bacteriology, vol. 188, no. 24, pp. 8360-8367, 2006.

[67] S. K. Christensen, M. Mikkelsen, K. Pedersen, and K. Gerdes, "RelE, a global inhibitor of translation, is activated during nutritional stress," Proceedings of the National Academy of Sciences of the United States of America, vol. 98, no. 25, pp. 14328-14333, 2001.

[68] C. Unoson and E. G. H. Wagner, "A small SOS-induced toxin is targeted against the inner membrane in Escherichia coli," Molecular Microbiology, vol. 70, no. 1, pp. 258-270, 2008.

[69] Y. Kim, X. Wang, X. S. Zhang et al., "Escherichia coli toxin/ antitoxin pair MqsR/MqsA regulate toxin CspD," Environmental Microbiology, vol. 12, no. 5, pp. 1105-1121, 2010. 
[70] M. G. Jørgensen, D. P. Pandey, M. Jaskolska, and K. Gerdes, "HicA of Escherichia coli defines a novel family of translationindependent mRNA interferases in bacteria and archaea," Journal of Bacteriology, vol. 191, no. 4, pp. 1191-1199, 2009.

[71] Y. Zhang, Y. Yamaguchi, and M. Inouye, "Characterization of YafO, an Escherichia coli toxin," Journal of Biological Chemistry, vol. 284, no. 38, pp. 25522-25531, 2009.

[72] K. K. Seok, B. Loll, T. C. Wai et al., "Molecular and structural characterization of the PezAT chromosomal toxin-antitoxin system of the human pathogen Streptococcus pneumoniae," Journal of Biological Chemistry, vol. 282, no. 27, pp. 1960619618, 2007.

[73] O. Pellegrini, N. Mathy, A. Gogos, L. Shapiro, and C. Condon, "The Bacillus subtilis ydcDE operon encodes an endoribonuclease of the MazF/PemK family and its inhibitor," Molecular Microbiology, vol. 56, no. 5, pp. 1139-1148, 2005.

[74] S. Agarwal, S. Agarwal, and R. Bhatnagar, "Identification and characterization of a novel toxin-antitoxin module from Bacillus anthracis," FEBS Letters, vol. 581, no. 9, pp. 1727 1734, 2007.

[75] D. P. Pandey and K. Gerdes, "Toxin-antitoxin loci are highly abundant in free-living but lost from host-associated prokaryotes," Nucleic Acids Research, vol. 33, no. 3, pp. 966976, 2005.

[76] L. Zhao and J. Zhang, "Biochemical characterization of a chromosomal toxin-antitoxin system in Mycobacterium tuberculosis," FEBS Letters, vol. 582, no. 5, pp. 710-714, 2008.

[77] J. Robson, J. L. McKenzie, R. Cursons, G. M. Cook, and V. L. Arcus, "The vapBC operon from Mycobacterium smegmatis is an autoregulated toxin-antitoxin module that controls growth via inhibition of translation," Journal of Molecular Biology, vol. 390, no. 3, pp. 353-367, 2009.

[78] L. Miallau, M. Faller, C. Janet et al., "Structure and proposed activity of a member of the VapBC family of toxin-antitoxin systems VapBC-5 from Mycobacterium tuberculosis," Journal of Biological Chemistry, vol. 284, no. 1, pp. 276-283, 2009.

[79] P. P. Budde, B. M. Davis, J. Yuan, and M. K. Waldor, "Characterization of a higBA toxin-antitoxin locus in Vibrio cholerae," Journal of Bacteriology, vol. 189, no. 2, pp. 491-500, 2007.

[80] R. A. F. Wozniak and M. K. Waldor, "A toxin-antitoxin system promotes the maintenance of an integrative conjugative element," PLoS Genetics, vol. 5, no. 3, Article ID e1000439, 2009.

[81] M. Cashel and K. E. Rudd, "The stringent response," in Escherichia coli and Salmonella typhimurium: Cellular and Molecular Biology, pp. 1410-1438, American Society for Microbiology, Washington, DC, USA, 1987.

[82] I. Kolodkin-Gal, R. Hazan, A. Gaathon, S. Carmeli, and H. Engelberg-Kulka, "A linear pentapeptide is a quorum-sensing factor required for mazEF-mediated cell death in Escherichia coli," Science, vol. 318, no. 5850, pp. 652-655, 2007.

[83] I. Kolodkin-Gal and H. Engelberg-Kulka, "The extracellular death factor: physiological and genetic factors influencing its production and response in Escherichia coli," Journal of Bacteriology, vol. 190, no. 9, pp. 3169-3175, 2008.

[84] H. Engelberg-Kulka, I. Yelin, and I. Kolodkin-Gal, "Activation of a built-in bacterial programmed cell death system as a novel mechanism of action of some antibiotics," Communitative and Integrative Biology, vol. 2, no. 3, pp. 211212, 2009.
[85] I. Kolodkin-Gal, B. Sat, A. Keshet, and H. Engelberg-Kulka, "The communication factor EDF and the toxin-antitoxin module mazEF determine the mode of action of antibiotics," PLoS Biology, vol. 6, no. 12, pp. 2774-2785, 2008.

[86] S. Amitai, I. Kolodkin-Gal, M. Hananya-Meltabashi, A. Sacher, and H. E. Kulka, "Escherichia coli MazF leads to the simultaneous selective synthesis of both "death proteins" and "survival proteins"," PLoS Genetics, vol. 5, no. 3, Article ID e1000390, 2009.

[87] W. C. Cheng and J. M. Hardwick, "A quorum on bacterial programmed cell death," Molecular Cell, vol. 28, no. 4, pp. 515-517, 2007.

[88] A. King and E. Gottlieb, "Glucose metabolism and programmed cell death: an evolutionary and mechanistic perspective," Current Opinion in Cell Biology, vol. 21, no. 6, pp. 885-893, 2009.

[89] M. E. Davey and G. A. O’Toole, "Microbial biofilms: from ecology to molecular genetics," Microbiology and Molecular Biology Reviews, vol. 64, no. 4, pp. 847-867, 2000.

[90] C. Sternberg, B. B. Christensen, T. Johansen et al., "Distribution of bacterial growth activity in flow-chamber biofilms," Applied and Environmental Microbiology, vol. 65, no. 9, pp. 4108-4117, 1999.

[91] S. A. Rani, B. Pitts, H. Beyenal et al., "Spatial patterns of DNA replication, protein synthesis, and oxygen concentration within bacterial biofilms reveal diverse physiological states," Journal of Bacteriology, vol. 189, no. 11, pp. 4223-4233, 2007.

[92] K. C. Rice, E. E. Mann, J. L. Endres et al., "The cidA murein hydrolase regulator contributes to DNA release and biofilm development in Staphylococcus aureus," Proceedings of the National Academy of Sciences of the United States of America, vol. 104, no. 19, pp. 8113-8118, 2007.

[93] J. S. Webb, L. S. Thompson, S. James et al., "Cell death in Pseudomonas aeruginosa biofilm development," Journal of Bacteriology, vol. 185, no. 15, pp. 4585-4592, 2003.

[94] S. A. West, S. P. Diggle, A. Buckling, A. Gardner, and A. S. Griffin, "The social lives of microbes," Annual Review of Ecology, Evolution, and Systematics, vol. 38, pp. 53-77, 2007.

[95] Y. Kim, X. Wang, Q. Ma, X. S. Zhang, and T. K. Wood, "Toxin-antitoxin systems in Escherichia coli influence biofilm formation through YjgK (TabA) and fimbriae," Journal of Bacteriology, vol. 191, no. 4, pp. 1258-1267, 2009.

[96] A. Mai-Prochnow, F. Evans, D. Dalisay-Saludes et al., "Biofilm development and cell death in the marine bacterium Pseudoalteromonas tunicata," Applied and Environmental Microbiology, vol. 70, no. 6, pp. 3232-3238, 2004.

[97] V. Tsilibaris, G. Maenhaut-Michel, N. Mine, and L. Van Melderen, "What is the benefit to Escherichia coli of having multiple toxin-antitoxin systems in its genome?" Journal of Bacteriology, vol. 189, no. 17, pp. 6101-6108, 2007.

[98] I. Kolodkin-Gal, R. Verdinger, A. Shlosberg-Fedida, and H. Engelberg-Kulka, "A differential effect of E. coli toxinantitoxin systems on cell death in liquid media and biofilm formation," PLoS One, vol. 4, no. 8, Article ID e6785, 2009.

[99] Y. Kim and T. K. Wood, "Toxins Hha and CspD and small RNA regulator $\mathrm{Hfq}$ are involved in persister cell formation through MqsR in Escherichia coli," Biochemical and Biophysical Research Communications, vol. 391, no. 1, pp. 209-213, 2010.

[100] I. Kolodkin-Gal and H. Engelberg-Kulka, "The stationaryphase sigma factor $\sigma$ is responsible for the resistance of Escherichia coli stationary-phase cells to mazEF-mediated cell death," Journal of Bacteriology, vol. 191, no. 9, pp. 3177-3182, 2009. 
[101] H. Engelberg-Kulka, B. Sat, M. Reches, S. Amitai, and R. Hazan, "Bacterial programmed cell death systems as targets for antibiotics," Trends in Microbiology, vol. 12, no. 2, pp. 6671, 2004.

[102] K. Pedersen, S. K. Christensen, and K. Gerdes, "Rapid induction and reversal of a bacteriostatic condition by controlled expression of toxins and antitoxins," Molecular Microbiology, vol. 45, no. 2, pp. 501-510, 2002.

[103] M. Christensen-Dalsgaard, M. G. Jørgensen, and K. Gerdes, "Three new RelE-homologous mRNA interferases of Escherichia coli differentially induced by environmental stresses," Molecular Microbiology, vol. 75, no. 2, pp. 333-348, 2010.

[104] I. Kolodkin-Gal and H. Engelberg-Kulka, "Induction of Escherichia coli chromosomal mazEF by stressful conditions causes an irreversible loss of viability," Journal of Bacteriology, vol. 188, no. 9, pp. 3420-3423, 2006.

[105] E. Diago-Navarro, A. M. Hernandez-Arriaga, J. LópezVillarejo et al., "parD toxin-antitoxin system of plasmid R1-basic contributions, biotechnological applications and relationships with closely-related toxin-antitoxin systems," FEBS Journal, vol. 277, no. 15, pp. 3097-3117, 2010.

[106] R. Díaz-Orejas, E. Diago-Navarro, A. M. H. Arriaga et al., "Bacterial toxin-antitoxin systems targeting translation," Journal of Applied Biomedicine, vol. 8, no. 4, pp. 179-188, 2010.

[107] A. A. Prozorov and V. N. Danilenko, "Toxin-antitoxin systems in bacteria: apoptotic tools or metabolic regulators?" Microbiology, vol. 79, no. 2, pp. 129-140, 2010.

[108] R. Jayaraman, "Bacterial persistence: some new insights into an old phenomenon," Journal of Biosciences, vol. 33, no. 5, pp. 795-805, 2008.

[109] R. D. Magnuson, "Hypothetical functions of toxin-antitoxin systems," Journal of Bacteriology, vol. 189, no. 17, pp. 60896092, 2007.

[110] L. Van Melderen and M. S. De Bast, "Bacterial toxin-antitoxin systems: more than selfish entities?” PLoS Genetics, vol. 5, no. 3, pp. 1-6, 2009.

[111] K. C. Rice and K. W. Bayles, "Molecular control of bacterial death and lysis," Microbiology and Molecular Biology Reviews, vol. 72, no. 1, pp. 85-109, 2008.

[112] S. Kornbluth and K. White, "Apoptosis in Drosophila: neither fish nor fowl (nor man, nor worm)," Journal of Cell Science, vol. 118, no. 9, pp. 1779-1787, 2005. 

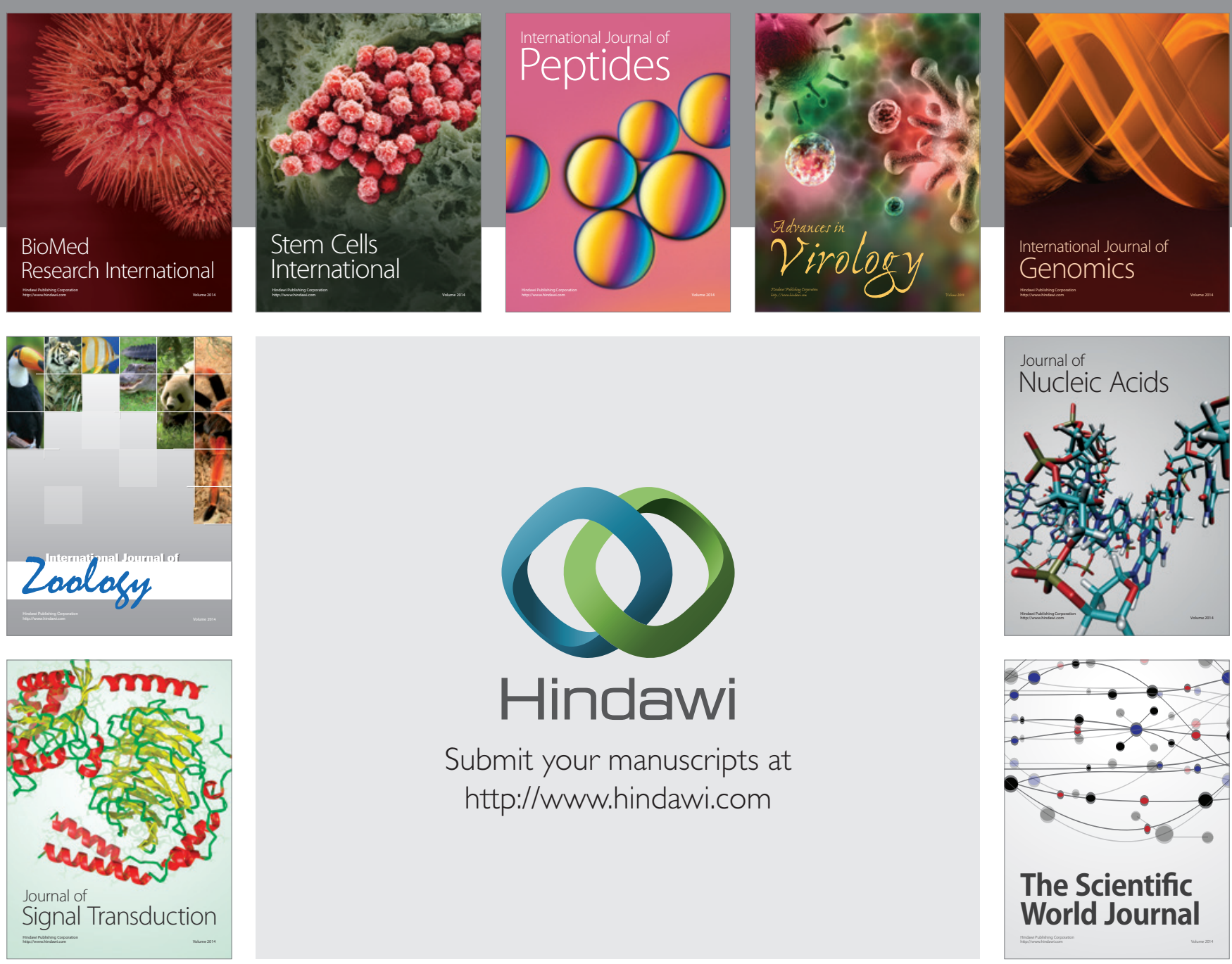

Submit your manuscripts at

http://www.hindawi.com
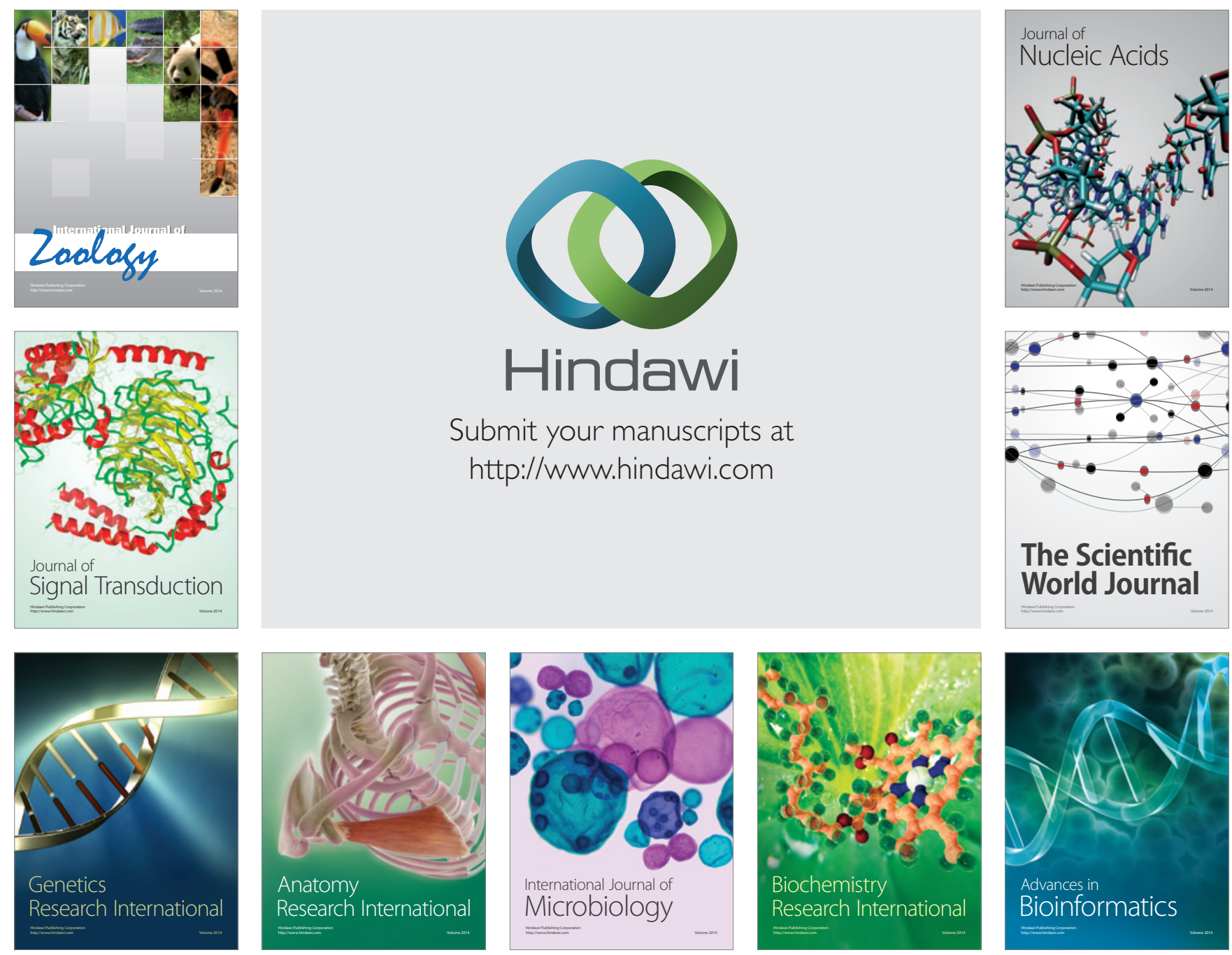

The Scientific World Journal
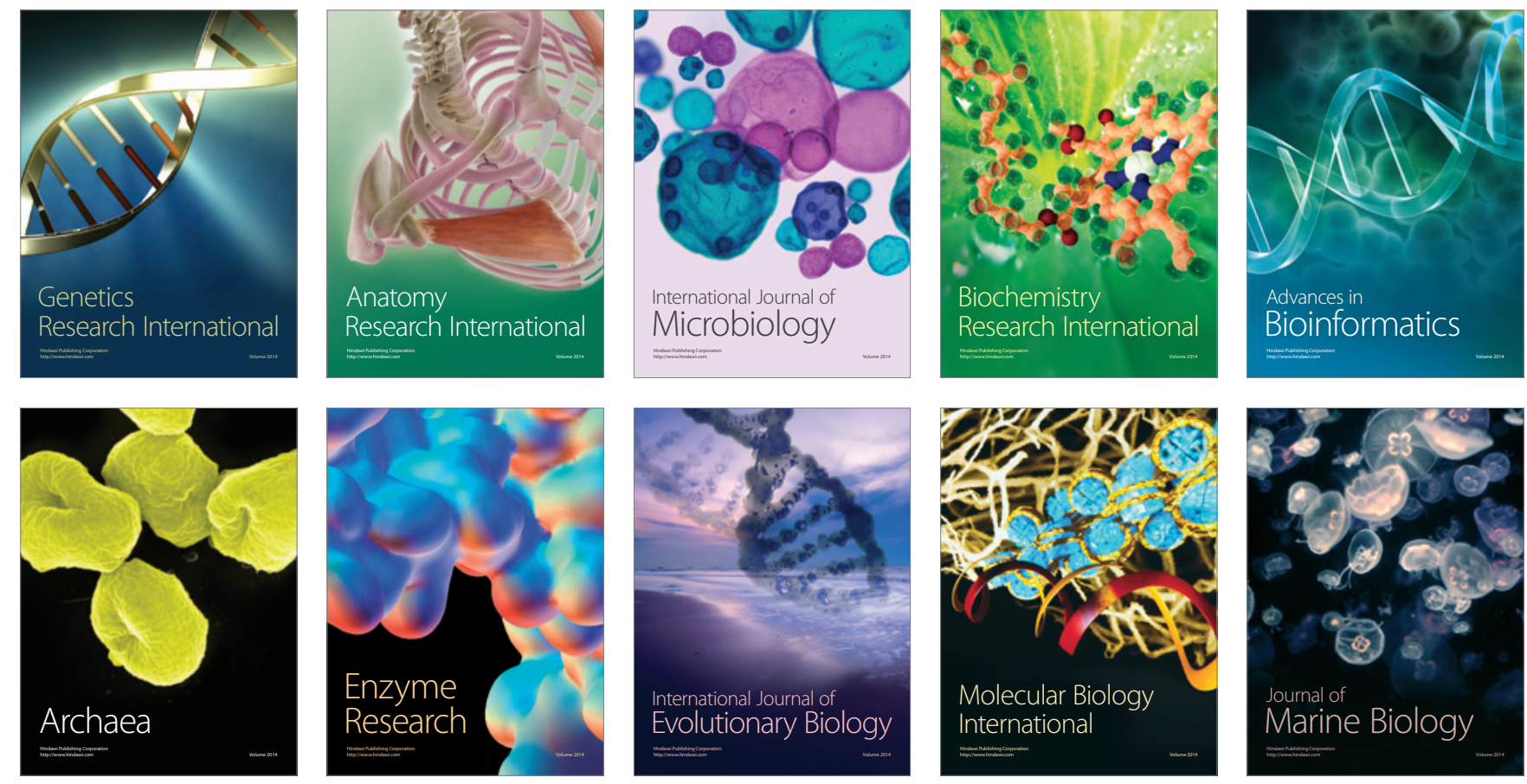